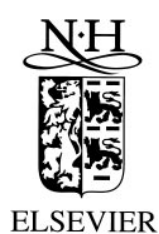

Surface Science 422 (1999) L177-L182

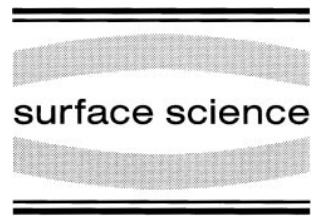

Surface Science Letters

\title{
A general rule for surface reconstructions of III-V semiconductors
}

\author{
S. Mirbt ${ }^{\mathrm{a}, *}$, N. Moll ${ }^{\mathrm{b}}$, A. Kley ${ }^{\mathrm{b}}$, J.D. Joannopoulos ${ }^{\mathrm{a}}$ \\ a Department of Physics, Massachusetts Institute of Technology, Cambridge, MA 02139, USA \\ ${ }^{\mathrm{b}}$ Fritz-Haber-Institut der Max-Planck-Gesellschaft, Faradayweg 4-6, D-14195 Berlin, Germany
}

Received 29 September 1998; accepted 10 November 1998

\begin{abstract}
First principles total energy calculations are performed for a large number (70) of III-V semiconductor surfaces in order to establish a database from which a general rule is extracted to help isolate and predict the lowest energy atomic surface geometries for these complex systems. The general rule involves minimizing a single, material- and geometry-independent, parameter, whose value depends only on a weighted sum of specific surface atom and bond structural units. (C) 1999 Elsevier Science B.V. All rights reserved.
\end{abstract}

Keywords: Density functional calculations; Low index single crystal surfaces; III-V semiconductors

The reconstructions and detailed atomic geometries of III-V semiconductor surfaces have been a subject of continuing interest over the past 20 years. A large number of experimental and theoretical studies [1] have led to the formulation of some general principles.

One general principle is the so-called electron counting rule [2], which is the 'sine qua non' for a surface to remain semiconducting. The cation (i.e. the Group III element) dangling bonds are significantly higher in energy than the anion (i.e. the Group V element) dangling bonds. Thus the cation dangling bonds tend to be empty, whereas the anion dangling bonds tend to be filled. This electron counting principle ensures that the

\footnotetext{
* Corresponding author. Present address: Condensed Matter Theory, Box 530, 75121 Uppsala, Sweden.

Fax: +46-18-4713524; e-mail: susanne@fysik.uu.se.
}

number of electrons matches the number of filled orbitals, thereby avoiding partially filled orbitals (i.e. the metallic state). Another general principle [1] is the formation of dimers on the surface, which leads to a reduction in the number of dangling bonds.

Although we find these principles to be operable in our database, they, in themselves, are not sufficient to help predict which of any two given surface geometries is lowest in energy. For example, in GaAs, as shown in Fig. 1, we find no correlation between the energies of a variety of surfaces and their associated numbers of As dangling bonds.

From an experimental point of view it is often desirable to know which of several suspected surface structures is lower in energy. This is because the experimental result typically provides only a fingerprint of the underlying surface structure. For example, scanning tunnel microscopy probes 


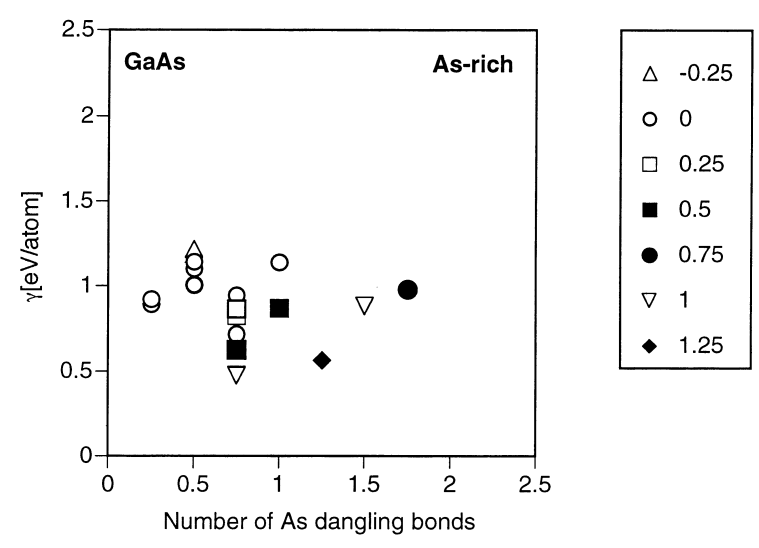

Fig. 1. GaAs surface energy in equilibrium with an anion-rich reservoir as a function of the number of As dangling bonds. The symbols indicate the stoichiometry of the corresponding surface reconstruction.

charge-density contours, which then have to be interpreted with the help of guessing some detailed atomic surface geometry. Theoretical calculations can help in this regard, but they also need to be guided by some simple physical rules which can a priori be used to identify the most promising candidates.

For example, one approach is to express the energy of a particular surface in terms of various bond and dangling-bond energy parameters that are fit to ab initio calculations [3]. This is an excellent method for exploring a variety of surface reconstructions for a given surface orientation of a given material. In this Letter we explore a different approach that is more qualitative in its predictive power but also much more general. Specifically, we introduce a single, material independent and surface-orientation independent parameter whose value depends only on the correct weighted sum of the appropriate surface atom and bond units, and whose evaluation requires no ab initio calculations. As we shall see, the smallest values of this parameter will generally correspond to the lowest surface energy geometries of any III-V material for any surface orientation. Consequently, a simple counting of specific surface atom and bond units associated with any given model III-V surface geometry is all that is needed to quickly isolate the lowest energy candidates.

We begin by creating an extensive database of surface energies using ab initio total energy calculations. This calculation technique is well known $[4,5]$ to give surface energies in very good agreement with experiment. Specifically, 70 surface geometries were calculated involving the (100), (110), (111), and ( $\overline{1} \overline{1} \overline{1})$ surfaces of InAs, InP, GaAs and AlAs. All semiconducting surface reconstruction geometries that are discussed in Refs. $[4,6]$ are included in the database. The surface energies were calculated using a density-functional theory [7] pseudopotential total energy approach [8]. The local-density approximation was applied to the exchange-correlation energy-functional [9] and the In, Ga, Al, As, and P atoms were described by fully separable norm-conserving ab initio pseudopotentials [10]. The wave functions were expanded in plane waves with an energy cutoff of $10 \mathrm{Ry}$. The electron density was calculated using special $\boldsymbol{k}$-point sets [11] with a density equivalent to $64 \boldsymbol{k}$-points in the entire $(100)(1 \times 1)$ surface Brillouin zone. To obtain the absolute surface energies for (111) and ( $\overline{1} \overline{1} \overline{1})$ orientations we employed the energy density formalism introduced by Chetty and Martin [12].

Using this database, we attempt to identify a simple relation between the surface energy $\gamma$ and the most important atomic structural elements that characterize a particular surface geometry. Different surface structures vary in the number per $(1 \times 1)$ surface unit cell of anion dangling bonds $N_{\mathrm{a}}$, cation dangling bonds $N_{\mathrm{c}}$, anion dimers $N_{\mathrm{a}-\mathrm{a}}$, and cation dimers $N_{\mathrm{c}-\mathrm{c}}$. The database confirms that the cation dangling bonds really are empty and, therefore, we assume that this contribution to the surface energy is negligible. Moreover, the database reveals that, in most cases, strain and stress contributions to the surface energy are of relatively minor importance. This is also in agreement with Duke [1], who reports strain and stress energies to be usually at least one order of magnitude smaller than bond energies. In an attempt to maintain a simple rule, we therefore neglect strain and stress. Moreover, we neglect the extended chemical environment of the atoms [3] and focus only on the local environment.

For a III-V semiconductor the surface energy $\gamma$ is a function not only of the surface geometry but also of the surface stoichiometry $\Delta N$, i.e. the 


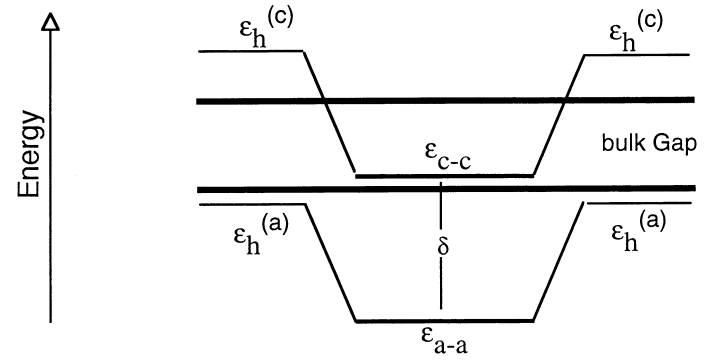

Fig. 2. Schematic energy level diagram showing the anion dimer energy level $\epsilon_{\mathrm{a}-\mathrm{a}}$, and cation dimer energy level $\epsilon_{\mathrm{c}-\mathrm{c}}$, in relation to the bulk band gap. The $\epsilon_{\mathrm{h}}$ indicate atomic $\mathrm{sp}^{3}$ hybrid energy levels. Also indicated is the correction term $\delta$. Note, that $\epsilon_{\mathrm{a}-\mathrm{a}}\left(\epsilon_{\mathrm{c}-\mathrm{c}}\right)$ is measured relative to $\epsilon_{\mathrm{h}}^{\mathrm{a}}\left(\epsilon_{\mathrm{h}}^{\mathrm{c}}\right)$.

difference in the number of atoms of the two species $i$. We assume the surface to be in equilibrium with a reservoir of the III- and V-species. In order to compare surfaces with different stoichiometries we allow the surface to exchange atoms with a reservoir characterized by a chemical potential $\mu_{i}^{1}$. and thereby adjust all surface energies to a common choice of zero. In equilibrium with a $\mathrm{V}$-rich (V-poor) reservoir a V-rich (V-poor) surface is energetically favored. Thus for every surface geometry the surface energy is bounded by two extreme cases, i.e. equilibrium with an anion-rich (AR) or a cation-rich (CR) reservoir.

Given these conditions, the surface energy of a crystal $\gamma$ can be approximated ${ }^{2}$ as the sum over the energies of all filled orbitals plus one correction term:

$$
\begin{aligned}
\gamma= & N_{\mathrm{a}-\mathrm{c}} \epsilon_{\mathrm{a}-\mathrm{c}}+N_{\mathrm{a}} \epsilon_{\mathrm{a}}+N_{\mathrm{a}-\mathrm{a}} \epsilon_{\mathrm{a}-\mathrm{a}}+N_{\mathrm{c}-\mathrm{c}}\left(\epsilon_{\mathrm{c}-\mathrm{c}}+\delta\right) \\
& -\mu_{\mathrm{c}} M_{\mathrm{c}}-\mu_{\mathrm{a}} M_{a} .
\end{aligned}
$$

Here $\epsilon_{\mathrm{a}}$ is the anion dangling bond energy and $\epsilon_{\mathrm{a}-\mathrm{a}}, \epsilon_{\mathrm{c}-\mathrm{c}}$ are dimer bond energies per atom, all relative to isolated atoms. For example, $\epsilon_{\mathrm{a}-\mathrm{a}}$ can be taken to be equal to the cohesive energy per atom of an anion crystal. In Fig. 2 we show a schematic energy level diagram. One realizes that the energy eigenvalue of the cation dimer lies higher in energy than the energy eigenvalue of the

\footnotetext{
${ }^{1}$ More details about the dependence of the surface energy on the chemical potential may be found in Ref. [4]

${ }^{2}$ Eq. (1) is derived by simply counting all bonds of the surface atoms.
}

anion dimer. Thus the correction term $\delta$ of Eq. (1) is equal to the difference between the cation dimer and anion dimer single particle eigenvalues with respect to their relative atomic energy levels, i.e. $\delta=\epsilon_{\mathrm{c}-\mathrm{c}}-\epsilon_{\mathrm{a}-\mathrm{a}}-\left(\epsilon_{\mathrm{h}}^{\mathrm{c}}-\epsilon_{\mathrm{h}}^{\mathrm{a}}\right)$ with $\epsilon_{\mathrm{h}}^{\mathrm{c}}$ and $\epsilon_{\mathrm{h}}^{\mathrm{a}}$ defined as in Fig. 2.

In this way, we account for the fact that at the surface the formation of a cation dimer is energetically more costly than the formation of an anion dimer. $M_{i}$ denotes the total number of particles of the species $i$.

In the following we assume the surface anioncation bond energy to be identical to its bulk value and thus rewrite Eq. (1) the following way:

$$
\begin{aligned}
\gamma= & N_{\mathrm{a}} \epsilon_{\mathrm{a}}+N_{\mathrm{a}-\mathrm{a}} \epsilon_{\mathrm{a}-\mathrm{a}}+N_{\mathrm{c}-\mathrm{c}}\left(\epsilon_{\mathrm{c}-\mathrm{c}}+\delta\right) \\
& -2 \mu_{\mathrm{c}} N_{\mathrm{c}-\mathrm{c}}-2 \mu_{\mathrm{a}} N_{\mathrm{a}-\mathrm{a}} .
\end{aligned}
$$

This is plausible, because of the following: In thermodynamic equilibrium the sum of chemical potentials of the anion and cation must be equal to the bulk energy per anion-cation pair

$\mu_{\mathrm{a}}+\mu_{\mathrm{c}}=\mu_{\mathrm{a}-\mathrm{c}}=2 \epsilon_{\mathrm{a}-\mathrm{c}}$.

Thus, $N_{\mathrm{a}-\mathrm{c}} \epsilon_{\mathrm{a}-\mathrm{c}}-\mu_{\mathrm{c}} M_{\mathrm{c}}-\mu_{\mathrm{a}} M_{\mathrm{a}}$ is approximately equal to $-2 \mu_{\mathrm{c}} N_{\mathrm{c}-\mathrm{c}}-2 \mu_{\mathrm{a}} N_{\mathrm{a}-\mathrm{a}}$, because all atoms that are exclusively bonded to an atom of the other species are no longer counted. Therefore, only dimer-bonded atoms remain to be counted and the factor of two enters, because every dimer bond consists of two atoms.

In order to proceed we will discuss Eq. (2) for the two extreme cases separately. In equilibrium with a $\mathrm{CR}$ reservoir we assume

$\mu_{\mathrm{a}}=0$ and $\mu_{\mathrm{c}}=\epsilon_{\mathrm{c}-\mathrm{c}}$.

This is certainly plausible because in the extreme case of equilibrium with a $\mathrm{CR}$ reservoir the cation chemical potential may be taken to be identical to the cation dimer bond energy $\epsilon_{\mathrm{c}-\mathrm{c}}$. The choice for $\mu_{\mathrm{a}}$ is arbitrary, but fixes henceforth our zero of energy because of Eq. (3).

If we now insert Eq. (4) into Eq. (2) we obtain

$\gamma=\epsilon_{\mathrm{a}} X_{\mathrm{CR}}$ 
with $X_{\mathrm{CR}}$ defined as

$X_{\mathrm{CR}}=N_{\mathrm{a}}+\frac{\epsilon_{\mathrm{a}-\mathrm{a}}}{\epsilon_{\mathrm{a}}} N_{\mathrm{a}-\mathrm{a}}+\frac{\delta-\epsilon_{\mathrm{c}-\mathrm{c}}}{\epsilon_{\mathrm{a}}} N_{\mathrm{c}-\mathrm{c}}$.

Eq. (5) predicts that the surface energy of any surface geometry of any given III-V semiconductor will scale linearly with $X_{\mathrm{CR}}$. Thus, $X_{\mathrm{CR}}$ identifies the most important surface structural elements and their corresponding weights. Surface geometries with the smallest $X_{\mathrm{CR}}$ should then yield the lowest surface energies. In order to test the general and practical nature of this hypothesis, it is very useful to simplify Eq. (6) so that it is material independent. Since we expect $\epsilon_{\mathrm{a}}$ is roughly on the order of $\epsilon_{\mathrm{a}-\mathrm{a}}$, we set

$\frac{\epsilon_{\mathrm{a}-\mathrm{a}}}{\epsilon_{\mathrm{a}}}=1$.

Similarly, using Harrison's tight binding results, ${ }^{3}$ we find it justified, to set

$\delta=\epsilon_{\mathrm{c}-\mathrm{c}}$.

With these approximations Eq. (6) now becomes

$X_{\mathrm{CR}}=N_{\mathrm{a}}+N_{\mathrm{a}-\mathrm{a}}$.

Thus the surface energy in equilibrium with a CR reservoir will be affected primarily by the number of anion dangling bonds and anion dimers. This is demonstrated in Fig. 3, where we plot our calculated CR surface energies $\gamma$ as a function of $X_{\mathrm{CR}}$ given by Eq. (9). We present results for four different materials: InAs, GaAs, InP, AlAs. Every data point corresponds to one first principles calculation for one specific surface orientation and surface geometry and the same symbol indicates the same stoichiometry as explained in Fig. 3. Note, that most of the data collapse nicely along a straight line, as predicted by Eqs. (5) and (9) for all of the materials studied. In addition, it is clear that stoichiometry alone cannot explain the most stable surface reconstructions.

\footnotetext{
${ }^{3}$ The cohesive energy of a cation crystal approximately equals $\delta$, which for GaAs may be obtained from the energy difference of $\mathrm{Ga}$ and $\mathrm{As} \mathrm{sp}^{3}$ orbitals. See for example the 'Table of Elements' in Ref. [13].
}

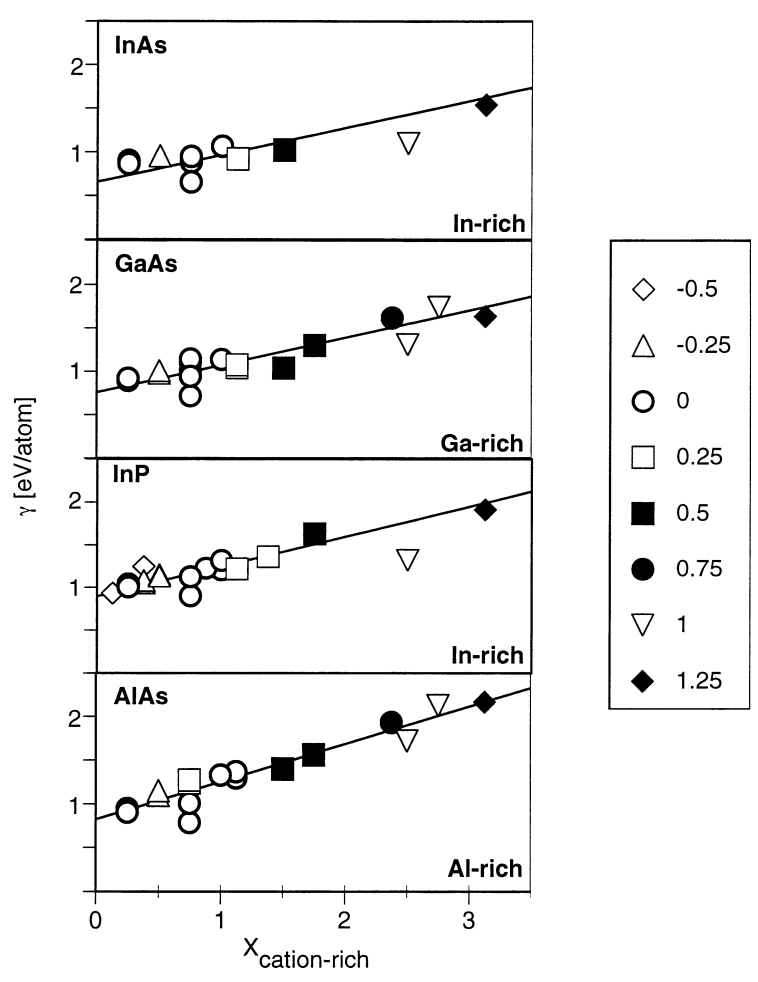

Fig. 3. Surface energies per atom in equilibrium with a CR reservoir as a function of $X_{\mathrm{CR}}$ [Eq. (9)], i.e. the sum of the number of anion dangling bonds and anion dimers per unit cell. The symbols indicate the stoichiometry of the corresponding surface reconstruction, i.e. for the (100) orientation the mixed $\operatorname{dimer}(2 \times 4)[\Delta N=-0.5]$, hexa $\operatorname{trimer}(2 \times 4)[\Delta N=-0.5]$, $\beta 2(4 \times 2)[\Delta N=-0.25], \beta(4 \times 2)[\Delta N=-0.25], \alpha(2 \times 4)[\Delta N=$ $0], \alpha(4 \times 2)[\Delta N=0], \delta(4 \times 2)[\Delta N=0], \quad \beta 2(2 \times 4)[\Delta N=0.25]$, $\beta(2 \times 4)[\Delta N=0.25], \quad \gamma(2 \times 4)[\Delta N=0.5], \quad \mathrm{c}(4 \times 4)-1 \mathrm{D}[\Delta N=$ $0.75], \mathrm{c}(4 \times 4)-2 \mathrm{D}[\Delta N=1]$, and $\mathrm{c}(4 \times 4)-3 \mathrm{D}[\Delta N=1.25]$; for the (110) orientation the cleavage $[\Delta N=0]$; for the (111) orientation the anion adatom $[\Delta N=0]$, anion $\operatorname{trimer}[\Delta N=0.5]$, and the

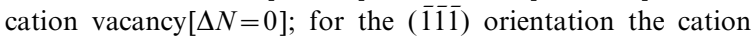
adatom $[\Delta N=0]$, anion vacancy $[\Delta N=0]$, and the anion trimer $[\Delta N=1]$.

The slopes of the lines in Fig. 3 are equal to the anion dangling bond energy [Eq. (5)] and are thus related to the bulk bonding energy. This is because the slope tells us how much bonding energy we lose by creating some particular surface. The slope is smallest for InAs $(0.31 \mathrm{eV} /$ atom $)$ followed by GaAs $(0.33 \mathrm{eV} /$ atom $)$ and $\operatorname{InP}(0.36 \mathrm{eV} /$ atom $)$. The largest slope is for AlAs $(0.43 \mathrm{eV} /$ atom $)$. These slopes are roughly proportional to the bulk bonding energies of InAs (3.1 eV/atom [13]), GaAs 
(3.26 eV/atom [13]), InP (3.48 eV/atom [13]) and AlAs (3.6 eV/atom [13]). From the approximation made in Eq. (7) we would expect identical slopes for InAs, GaAs, and AlAs. On the other hand, with $\epsilon_{\mathrm{a}-\mathrm{a}} / \epsilon_{\mathrm{a}}$ varying between 0.9 and 1.1 the $\epsilon_{\mathrm{a}}$-ratios are in agreement with the bulk bonding energy ratios. The approximations made earlier are thus roughly confirmed.

We now turn our attention to surfaces in equilibrium with an AR reservoir. In analogy to Eq. (4) we make the approximation

$\mu_{\mathrm{a}}=\epsilon_{\mathrm{a}-\mathrm{a}}$.

Because of our choice of zero and Eq. (3) we find

$\mu_{\mathrm{c}}=\epsilon_{\mathrm{c}-\mathrm{c}}-\epsilon_{\mathrm{a}-\mathrm{a}}$.

Insertion of Eqs. (10) and (11) into Eq. (2) gives

$\gamma=\epsilon_{\mathrm{a}} X_{\mathrm{AR}}$

with $X_{\mathrm{AR}}$ defined as

$X_{\mathrm{AR}}=N_{\mathrm{a}}-\frac{\epsilon_{\mathrm{a}-\mathrm{a}}}{\epsilon_{\mathrm{a}}} N_{\mathrm{a}-\mathrm{a}}+\frac{2 \epsilon_{\mathrm{a}-\mathrm{a}}+\delta-\epsilon_{\mathrm{c}-\mathrm{c}}}{\epsilon_{\mathrm{a}}} N_{\mathrm{c}-\mathrm{c}}$.

We again use the approximations of Eqs. (7) and (8) and then finally obtain

$X_{\mathrm{AR}}=N_{\mathrm{a}}-N_{\mathrm{a}-\mathrm{a}}+2 N_{\mathrm{c}-\mathrm{c}}$

Note that $N_{\mathrm{a}}$ appears both in Eqs. (9) and (14). This simply reflects the loss of bonding energy upon the formation of filled dangling bonds, since these two electrons can no longer participate in any bonding. In Fig. 4 we plot our calculated AR surface energies as a function of $X_{\mathrm{AR}}$ given by Eq. (14). Again we present results for four different materials: InAs, GaAs, InP, AlAs.

Again most of the data collapse nicely on a straight line. Comparing the slopes of Fig. 4 with those of Fig. 3, which should be identical, it turns out that the difference in slope is less than 5\%. This is another justification for the approximations made in Eqs. (7) and (8).

For the AR extreme case we note the surface energy becoming negative for AR surfaces. This is in accordance with Eq. (1), where assuming a surface with only anion dimers, the surface energy

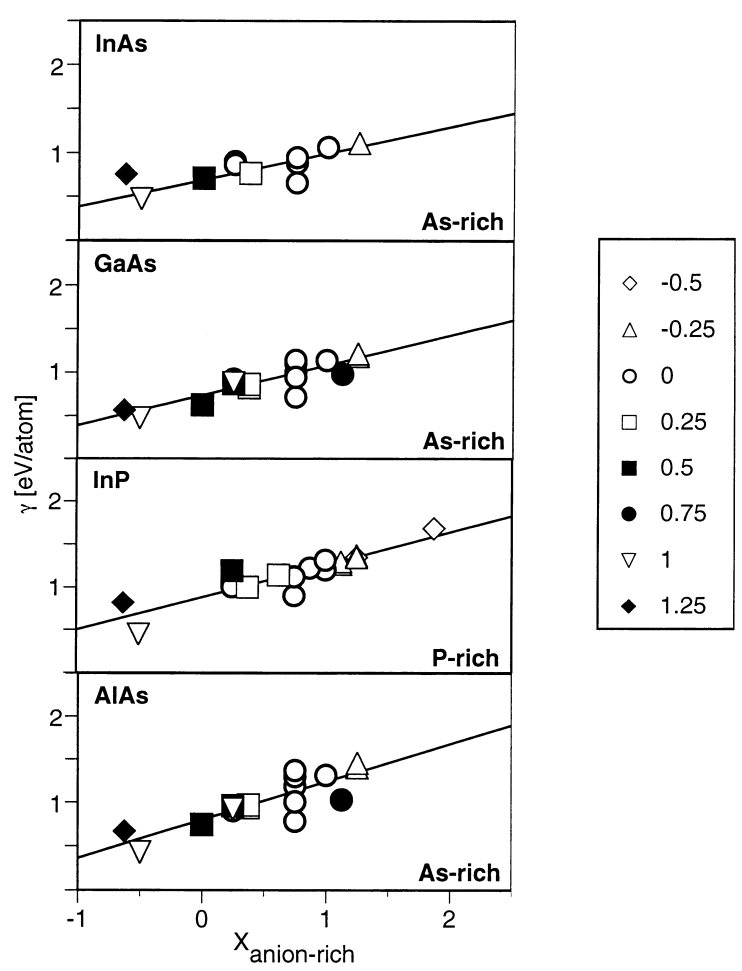

Fig. 4. Same as in Fig. 3, but surface energies are shown in equilibrium with an $\mathrm{AR}$ reservoir as a function of $X_{\mathrm{AR}}$ [Eq. (14)].

becomes

$\gamma=-N_{\mathrm{a}-\mathrm{a}} \epsilon_{\mathrm{a}-\mathrm{a}}$.

On the other hand, in the CR extreme case, assuming a surface with only cation dimers, the surface energy equals zero, which is consistent with our findings. Moreover, we find in Figs. 3 and 4 that the surface energy has some positive value for $X_{\mu}=0$, where instead our general rules predict it to become zero. This offset is also understood, considering the choice of zero [Eq. (4)] inherent in our model, which differs from the choice of zero in our database.

In the case of a general chemical potential $\mu$ we can simply interpolate between the two extreme cases treated above and obtain

$$
X_{\mu}=N_{\mathrm{a}}+\left(\frac{2 \mu-\mu_{\mathrm{CR}}}{\mu_{\mathrm{CR}}}\right) N_{\mathrm{a}-\mathrm{a}}+2\left(\frac{\mu_{\mathrm{CR}}-\mu}{\mu_{\mathrm{CR}}}\right) N_{\mathrm{c}-\mathrm{c}} .
$$


In this expression $\mu$ varies between $\mu_{\mathrm{CR}}$ and $\mu_{\mathrm{AR}}$, with $\mu_{\mathrm{AR}}$ being defined as zero.

In order to illustrate the applicability of our new rule, let us consider two examples. First, on GaAs (100), experiments reveal in the AR regime a $(4 \times 4)$ reconstruction which theoretically is believed to be the $\mathrm{c}(4 \times 4)-3 \mathrm{D}$ reconstruction with three anion dimers per unit cell. It includes ten anion dangling bonds and 15 anion dimers per unit cell, thus leading to $X_{\mathrm{AR}}=-0.625$. Two further $\mathrm{c}(4 \times 4)$ reconstructions were proposed, one with two anion dimers $\left(X_{\mathrm{AR}}=0.25\right)$, the other with one anion dimer $\left(X_{\mathrm{AR}}=1.125\right)$. Our general rule thus predicts, in agreement with both experiments and calculations, that the $\mathrm{c}(4 \times 4)-3 \mathrm{D}$ reconstruction should be energetically most favorable in the AR regime. We note that a $\mathrm{c}(4 \times 4)-4 \mathrm{D}$ reconstruction with four anion dimers $\left(X_{\mathrm{AR}}=-1.5\right)$ does not fulfill the electron counting rule. Therefore, it is not semiconducting and it is higher in energy.

Second, in the CR regime a trimer reconstruction was found on the (100) InP surface, which could not be understood within the context of known GaAs reconstruction models. Searching for surface geometries with the lowest possible value of $X_{\mathrm{CR}}$, we examined a novel reconstruction proposed by Schmidt and Bechstedt [14] with a value of $X_{\mathrm{CR}}=0.125$ and found that it corresponds to the lowest value of $X_{\mathrm{CR}}$ of any other geometry. We also calculated its total energy and confirmed that it corresponds to the lowest energy surface reconstruction.

In summary, the importance of our simple rule is that it allows us to isolate the lowest energy candidates for any III-V semiconducting surface of any orientation for a given chemical potential. It has not been possible, to date, to estimate the relative surface energy in such a simple way. With our rule a simple counting of the number of anion dangling bonds, anion dimers and cation dimers leads to an $X$, the value of which depends on the chemical potential. The structure with the lower $X$ is, in general, energetically favored over the structure with the larger $X$. Exceptions from this simple rule are mainly due to the neglect of strain and stress. Of course, better approximations may lead to a more complex set of rules, but our focus here was to attempt to identify a simple rule containing the most important physics that would be robust over a large database of materials, geometries and surface orientations.

\section{Acknowledgement}

S.M. is grateful to the Swedish Natural Science Research Council for financial support.

\section{References}

[1] C.B. Duke, Chem. Rev. 96 (1996) 1237.

[2] W.A. Harrison, J. Vac. Sci. Technol. 16 (1979) 1492.

[3] S.B. Zhang, A. Zunger, Phys. Rev. B 53 (1996) 1343.

[4] N. Moll, A. Kley, E. Pehlke, M. Scheffler, Phys. Rev. B 54 (1996) 8844.

[5] D.K. Biegelsen, R.D. Bringans, J.E. Northrup, L.E. Swartz, Phys. Rev. Lett. 65 (1990) 452.

[6] A. Kley, Ph.D. Thesis, Technical University of Berlin, 1997.

[7] W. Kohn, L.J. Sham, Phys. Rev. 140 (1965) A1133.

[8] R. Stumpf, M. Scheffler, Comput. Phys. Commun. 79 (1994) 447.

[9] J.P. Perdew, A. Zunger, Phys. Rev. B 23 (1981) 5048.

[10] D.R. Hamann, Phys. Rev. B 40 (1989) 2980.

[11] H.J. Monkhorst, J.D. Pack, Phys. Rev. B 13 (1976) 5188.

[12] N. Chetty, R.M. Martin, Phys. Rev. B 45 (1992) 6089.

[13] W.A. Harrison, Electronic Structure, Freeman Press, San Francisco, 1980.

[14] W.C. Schmidt, F. Bechstedt, Surf. Sci. 409 (1998) 474. 